\title{
Adaptive Equalization in Oversampled Subbands
}

\author{
S. Weiß ${ }^{\dagger}$, S.R. Dooley, R.W. Stewart, and A.K. Nandi
}

Signal Processing Division, Department of Electronic and Electrical Engineering University of Strathclyde, Glasgow G1 1XW

\begin{abstract}
The potential presence of fractional delays, non-minimum phase parts, and a colouring of the channel output can require adaptive equalizers to adapt very long filters, which can have slow convergence for LMS-type adaptive algorithms. This paper presents a novel oversampled subband approach to adaptive equalization, which can both significantly reduce computational complexity, and improve convergence speed.
\end{abstract}

\section{INTRODUCTION}

When data is transmitted through a channel, the characteristics of the channel generally create a signal distortion which may cause bit errors on the receiving side. Thus, in communication systems a filter is employed to equalize any linear distortions of the channel [1]. Similarly, audio systems often require equalization [2]. Usually an adaptive solution is preferred to account for time-varying behaviour.

Some types of characteristics are particularly hard to equalize, for example nonminimum phase systems [2, 3], and fractional delays [4], which involve the identification of very long and generally non-causal impulse responses. For adaptive equalization operating in the fullband, adaptive algorithms with high computational complexity such as the RLS cannot be used, while for computationally less complex LMS-type algorithms the convergence speed depends on the filter length. The latter is further slowed down when the channel introduces a severe amplitude distortion, since the input to the adaptive filter will be highly coloured. Therefore, the application of subband adaptive filters (SAF) appears sensible: by operating in decimated frequency bands as shown in Fig. 1, both the update rate and the length of the

\footnotetext{
${ }^{\dagger}$ POC: Dipl.-Ing. Stephan Weiss, Signal Processing Division, Dept. of Electronic and Electrical Engineering, University of Strathclyde, Glasgow G1 1XW, Scotland. Tel. 0044-141-548-2684, Fax 0044-141-552-2487; e-mail weiss@spd.eee.strath.ac.uk.
} 
adaptive filters can be greatly reduced leading to a lower computational complexity. Further, the subband decomposition performs a whitening of the input signal, resulting in improved convergence behaviour.

\section{ADAPTIVE FILTERING IN SUBBANDS}

In an SAF system, the subband decomposition is performed by filter banks (Fig. 2), producing $K$ subband signals decimated by a factor $N \leq K$. Critically decimated SAF systems (i.e. $N=K$ ) require either adaptive cross-terms between at least adjacent subbands [5], or the use of gap-filter banks with spectral loss to avoid any aliasing problems [6]. This motivates the use of oversampled SAF systems: while the decimation $N<K$ of real valued bandpass signals is problematic, and requires either modulation into the baseband prior to decimation [7] or the use of non-uniform filter banks [8], downsampling of complex valued bandpass signals is straightforward. In particular complex valued filter banks derived by generalized DFT modulation from a prototype filter are advantageous since they offer near perfect reconstruction and a computationally inexpensive implementation [7].

For an SAF system to perform satisfactorily, a delay in the path of the desired signal is required to account for various decimated transients of the analysis filter banks. If the SAF length is sufficient, no model truncation occurs, and if observation noise is absent, the minimum mean square error (MMSE) is limited only by the aliasing level produced by the decimation in the analysis filter banks, and the maximally achievable accuracy of the equalizer bound by the distortion function of the filter bank. In the case of modulated filter banks, both errors can be stated in terms of the prototype filter [9].

\section{SUBBAND ADAPTIVE EQUALIZATION}

A simple structure for an equalizer in subbands was introduced in Fig. 1, where the SAF system is applied in series to the distorting channel. For successful equalization, an adaptive algorithm is expected to perform an inverse system identification of the channel [3]. This becomes awkward as many real world channels are non-minimum phase systems, i.e. posses zeros outside the unit circle, e.g. in room acoustics [2] or communication systems [4]. Considering a maximum phase zero location $a,|a|>1$, 
the inverse system can be stated by two representations for FIR equalizers, obtained by polynomial division:

$$
\frac{1}{1+a z^{-1}}= \begin{cases}\sum_{i=0}^{\infty} a^{i} z^{-i} & \text { causal, unstable } \\ \frac{1}{a z^{-1}} \frac{1}{1+a^{-1} z}=\sum_{i=1}^{\infty} a^{-i} z^{i} & \text { non-causal, stable }\end{cases}
$$

Only the second solution is viable, and can be made partially causal by introducing a delay, which should be approximately half the length of the SAFs [3]. If the zero position $a$ is close to the unit circle, the impulse response of the inverse system can become very long. Furthermore, the channel generally colours the signal $x[n]$.

When performing adaptive equalization in oversampled complex valued subbands, the adaptive filter lengths can be chosen shorter in accordance with the sampling rate decimated by a factor $N<K$. Also updating now occurs at the lower rate. Following the approach in $[7,9]$, for real valued signals $x[n]$ and $d[n]$ only $K / 2$ complex subbands decimated by $N<K$ need to be processed, since the remaining subbands are complex conjugate and therefore redundant. This yields a reduction in computational complexity by a factor $r=\frac{K}{2 N^{2}}$ for LMS-type algorithms (omitting any additional costs introduced by the filter banks).

\section{APPLICATION EXAMPLE}

To demonstrate the benefit of adaptive equalization in subbands, a bandpass channel with magnitude and a non-linear phase response as shown Fig. 3(a) is used for simulation. Noise interference with spectral characteristics similar to the channel has been added at 40dB SNR. To invert this system, we apply an NLMS adaptive algorithm to both a 1500 tap fullband filter and an SAF system with $K / 2=16$ complex subbands (with bandedges indicated by vertical dashed lines in Fig. 3(a)), decimated by $N=28$ and having $(1500+896) / N \approx 86$ tap filters in each channel. The increase in length by $L_{h}=896$ is to approximately compensate for transients caused by the filter banks with an overall delay of $L_{h}$ samples. Both fullband and subband adaptive algorithms operate with the same normalized LMS step size [3]. From the ensemble mean squared error curves in Fig. 3(b), a clear advantage in adaptation speed for the SAF equalization system is evident, while also requiring only $22 \%$ of the computational resources of the fullband equalizer. 


\section{CONCLUSIONS}

We have motivated the application of subband adaptive filtering to the equalization problem, which can otherwise require a considerable filter length and suffer from slow convergence in a fullband implementation. The subband approach presented here used oversampled, near perfect reconstructing filter banks, which gives a significant reduction in computations and improved convergence speed over the fullband scheme.

\section{ACKNOWLEDGEMENTS}

The work of SW and RWS was supported by a scholarship from William Garven Research Bequest, Glasgow. SRD and AKN would like to thank the Engineering and Physical Sciences Research Council for their support.

\section{REFERENCES}

[1] PROAKIS, J. G.: 'Channel Equalization', in J. D. Gibson, ed., The Mobile Communications Handbook, Chap. 6, pp.56-80, CRC Press / IEEE Press, 1996.

[2] NEELY, S. T., and ALLEN, J. B.: 'Invertibility of a Room Impulse Response'. J Acoustic Soc. America, 1979, 66, (1), pp.165-169.

[3] WIDROW, B., and STEARNS, S. D.: Adaptive Signal Processing, Prentice Hall, Englewood Cliffs, NY, 1985.

[4] LAAKSO, T. I., VÄLIMÄKI, V., KARJALAINEN, M., and LAINE, U. K.: 'Splitting the Unit Delay'. IEEE Signal Process. Magazine, 1996, 13, (1), pp.3060 .

[5] GILloire, A., and VETTERLI, M.: 'Adaptive Filtering in Subbands with Critical Sampling: Analysis, Experiments and Applications to Acoustic Echo Cancelation', IEEE Trans. Signal Process., 1992, SP-44, (8), pp.1862-1875.

[6] SOMAYAZULU, V., MITRA, S., and SHYNK, J. J.: 'Adaptive Line Enhancement Using Multirate Techniques', in Proc. IEEE Intern. Conf. Acoustics, Speech, and Signal Process., 1989, 2, pp.928-931, Glasgow, Scotland, UK. 
[7] WEISS, S., LAMPE, L., and STEWART, R. W.: 'Efficient Implementations of Complex and Real Valued Filter Banks for Comparative Subband Processing with an Application to Adaptive Filtering.', in Proc. Intern. Symp. Comm. Systems Digit. Signal Process., 1998, pp.32-35, Sheffield, UK.

[8] HARTEnECK, M., PÁEZ-BORRAllo, J. M., and STEWART, R. W.: 'An Oversampled Subband Adaptive Filter Without Cross Adaptive Filters', Signal Process. , 1998, 64, (1), pp.93-101.

[9] WEisS, S., STEWART, R. W., STEnGER, A., and RABEnSTEIN, R.: 'Performance Limitations of Subband Adaptive Filters', to be pres. Europ. Signal Process. Conf., 1998, Rhodos, Greece.

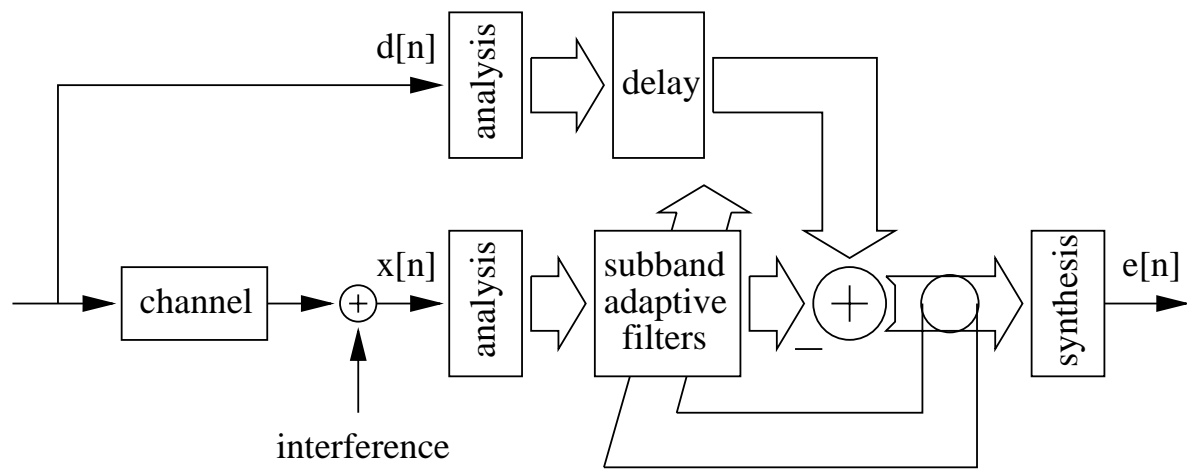

Fig. 1: Adaptive equalization in subbands.

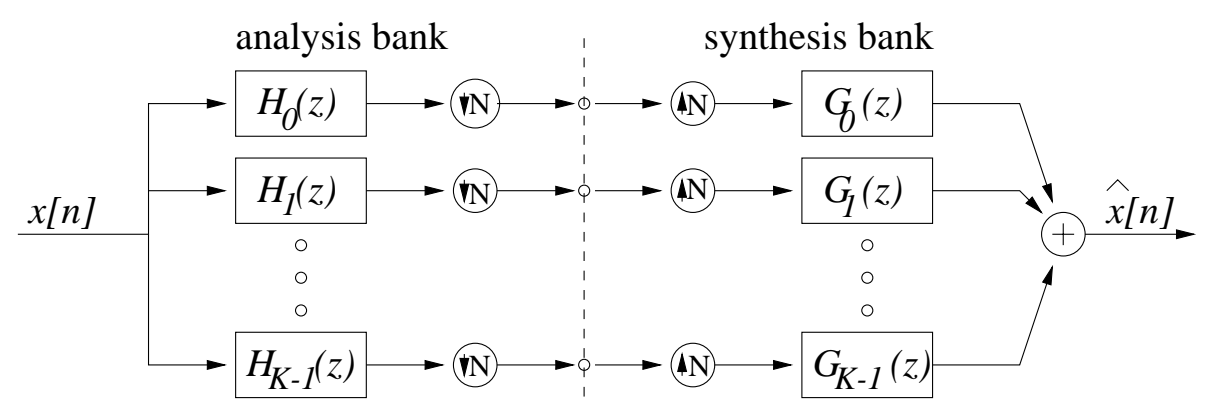

Fig. 2: Decomposition of a signal $x[n]$ by an analysis bank into $K$ subbands decimated by $N \leq K$; a fullband signal $\hat{x}[n]$ can be reconstructed by a synthesis bank. 
(a) channel characteristics
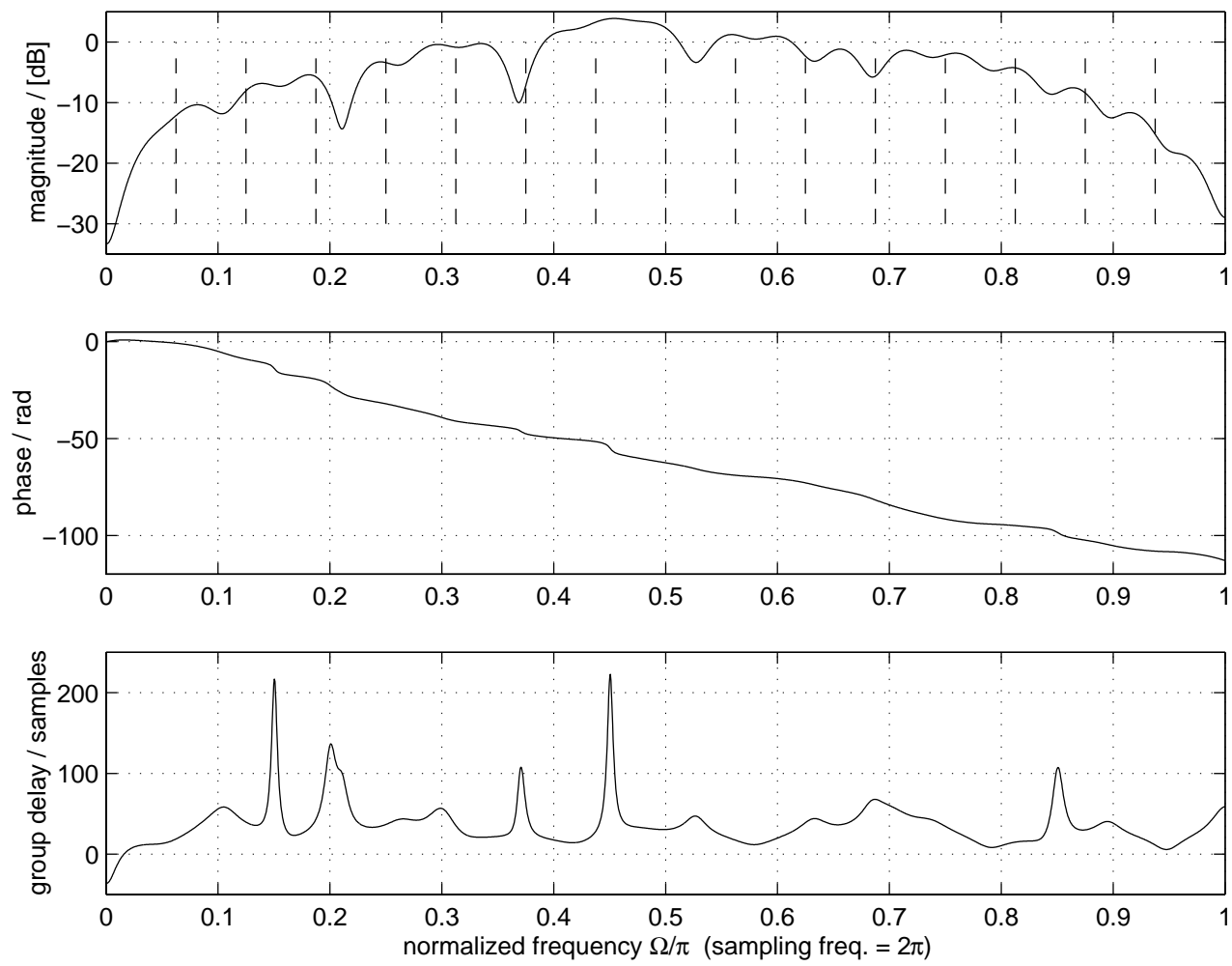

(b) MSE curves for adaptive equalization

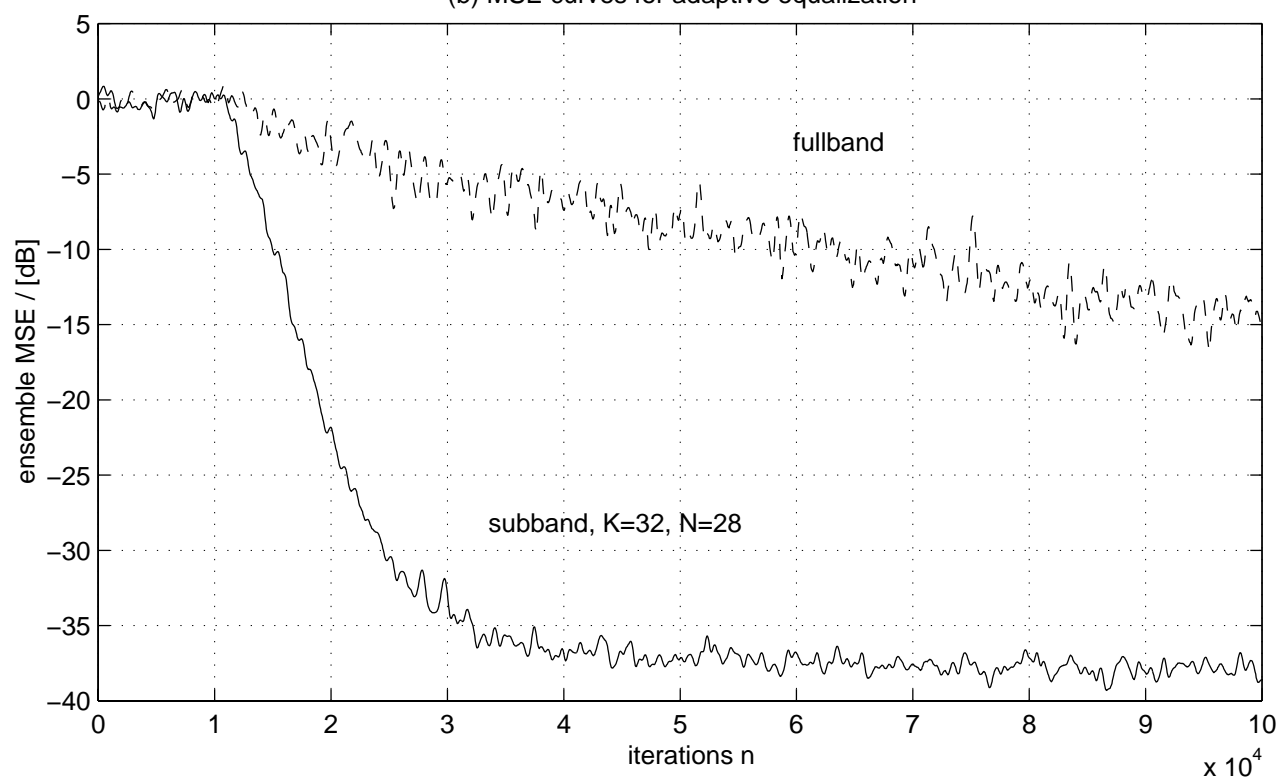

Fig. 3: (a) Magnitude, phase response and group delay of the channel; (b) ensemble MSE curves of SAF and fullband equalizers (adaptation switched on at $n=10^{4}$ ). 